\title{
Role of transport band edge variation on delocalized charge transport in high-mobility crystalline organic semiconductors
}

\author{
Andrey Kadashchuk,,${ }^{1,2, *}$ Fei Tong, ${ }^{3}$ Robby Janneck,,${ }^{1,4}$ Ivan I. Fishchuk, ${ }^{2,5}$ Alexander Mityashin, ${ }^{1}$ Egon Pavlica, ${ }^{3}$ \\ Anna Köhler, ${ }^{6}$ Paul Heremans, ${ }^{1,4}$ Cedric Rolin, ${ }^{1}$ Gvido Bratina, ${ }^{3}$ and Jan Genoe ${ }^{1,4}$ \\ ${ }^{1}$ IMEC, Kapeldreef 75, 3001 Leuven, Belgium \\ ${ }^{2}$ Institute of Physics, National Academy of Sciences of Ukraine, Prospect Nauky 46, 03028 Kyiv, Ukraine \\ ${ }^{3}$ Laboratory of Organic Matter Physics, University of Nova Gorica, Vipavska 13, Nova Gorica, SI-5000, Slovenia \\ ${ }^{4}$ KULeuven, ESAT, Kasteelpark Arenberg 10, 3001 Leuven, Belgium \\ ${ }_{5}^{5}$ Institute for Nuclear Research, National Academy of Sciences of Ukraine, Prospect Nauky 47, 03680 Kyiv, Ukraine \\ ${ }^{6}$ Experimental Physics II and Bayreuth Institute of Macromolecular Research (BIMF), Universitätsstraße 30, 95448 Bayreuth, Germany
}

(Received 7 March 2017; revised manuscript received 11 August 2017; published 12 September 2017)

\begin{abstract}
We demonstrate that the degree of charge delocalization has a strong impact on polarization energy and thereby on the position of the transport band edge in organic semiconductors. This gives rise to long-range potential fluctuations, which govern the electronic transport through delocalized states in organic crystalline layers. This concept is employed to formulate an analytic model that explains a negative field dependence coupled with a positive temperature dependence of the charge mobility observed by a lateral time-of-flight technique in a high-mobility crystalline organic layer. This has important implications for the further understanding of the charge transport via delocalized states in organic semiconductors.
\end{abstract}

DOI: 10.1103/PhysRevB.96.125202

\section{INTRODUCTION}

The improvement of electronic transport in organic semiconductors (OSs) is critical for a range of electronic applications, notably, for organic field-effect transistors (OFETs). There has been tremendous progress in developing both alternative OSs and fabrication techniques for thin crystalline films, which provide high charge-carrier mobilities $(\mu)$ well above $1 \mathrm{~cm}^{2} / \mathrm{V} \mathrm{s}$ [1-5]. At the same time, the fundamentals of the charge transport in OS films with charge mobilities in the range $\sim 1-10 \mathrm{~cm}^{2} / \mathrm{V} \mathrm{s}$ (relevant for many advanced OFETs) are still not completely understood. This mobility range falls into an intermediate region between two wellestablished transport regimes, namely, the thermally activated hopping transport applicable to low-mobility disordered OS and the band-transport regime observed in perfect highmobility organic single crystals. Understanding the processes that determine charge transport in this intermediate regime remains a challenge [6].

The dependence of the charge-carrier mobility on temperature $\mu(T)$ and electric field $\mu(E)$ are commonly used to elucidate the mechanism of charge transport. Disordered films of OSs with hopping charge transport typically show $\mu$ increasing with electric field in a Poole-Frenkel fashion $[\ln (\mu) \propto \sqrt{E}]$ and positive temperature dependence $(d \mu / d T>0)[7,8]$. On the contrary, in perfect organic single crystals, $\mu$ is normally independent of electric field at not-too-low temperatures $[9,10]$ and features a negative temperature dependence $(d \mu / d T<0)$ that is usually rationalized in terms of band motion. Despite great technological relevance of high-mobility crystalline OS thin films prepared from solution or by vacuum evaporation, their molecular ordering over the whole transistor channel might not necessarily be as perfect as in a high-quality bulk single crystal. Therefore, the charge transport mechanism in these films might differ from classical band transport.

*kadash@imec.be
In this paper, we report that energy levels in OSs vary significantly with the degree of charge delocalization. Hence the spatial distribution of the charge delocalization length can cause long-range potential fluctuations of the band edge in crystalline OS films. These fluctuations can strongly affect the delocalized charge transport within the highly crystalline, yet imperfect, organic film when the localized states do not play a dominant role. To validate our model, we fabricated highly crystalline films of 2,7-dioctyl[1]benzothieno[3,2-b][1]benzothiophene $\left(\mathrm{C}_{8}\right.$-BTBT $)$ - a renowned band-transport OS with a firmly established two-dimensional (2D) delocalization nature of transporting states [11,12]. Time-of-flight (TOF) photocurrent measurements of these films in a lateral transport configuration attested to $\mu$ as large as $\sim 20 \mathrm{~cm}^{2} / \mathrm{V} \mathrm{s}$, yet have surprisingly showed a negative $\mu(E)$ dependence at relatively high temperatures $(T \geqslant 295 \mathrm{~K})$ along with a thermally activated charge transport. This is unusual since this type of delocalized transport cannot be rationalized by classical band-type transport models. Here we show that this behavior can naturally be described by an analytic effective-medium approximation (EMA) model employing the concept of the transport band edge variations and notion of the field-dependent effective dimensionality of charge percolative paths.

\section{MOLECULAR MICROELECTROSTATIC MODEL CALCULATIONS}

We begin by assessing the impact of spatial variation of the charge delocalization length on energetic disorder within imperfect crystalline layers of OSs. Indeed, the conventional pointlike charge approximation can become problematic when states are extended, as was recently shown for, e.g., the Förster-type resonant energy transfer of delocalized excitons [13], and we believe that similar reasoning applies to delocalized charges. We calculated the charge transport energies in OS as a function of the degree of charge-carrier delocalization by means of a molecular microelectrostatic 

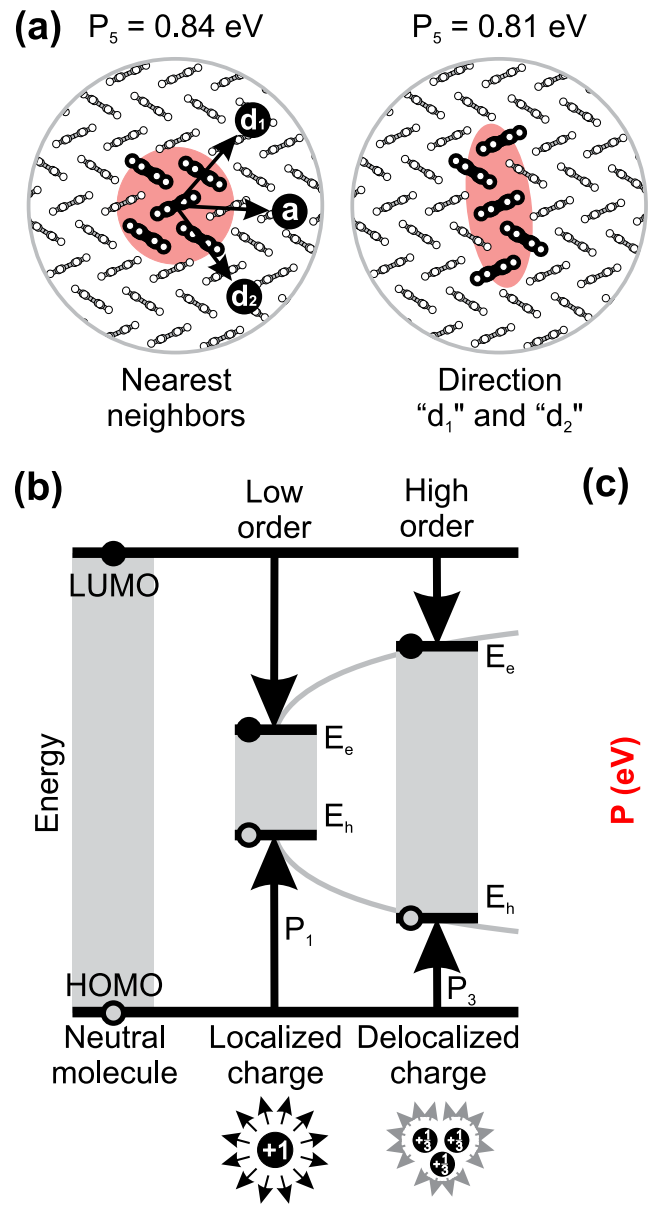

(c)
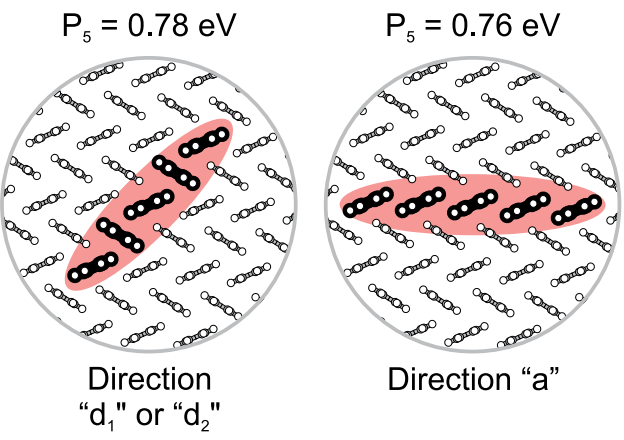

Direction "a"

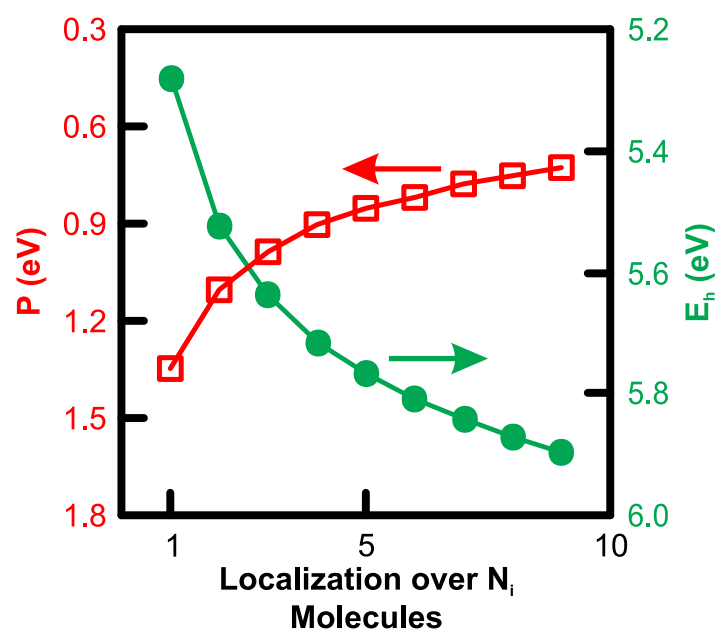

FIG. 1. Evolution of the electronic structure upon charge delocalization for the case of a monolayer of pentacene molecules. (a) Possible delocalization geometries in a monolayer of pentacene; for each case, we consider delocalization of a positive charge over $N=5$ molecules and tabulate the corresponding polarization energy $P$. (b) Sketch of the charge delocalization impact on the electron and hole transport band energies. (c) As the charge delocalization expands over more molecules, $P$ decreases; correspondingly, the hole transport energy $E_{h}$ approaches the HOMO level of a neutral pentacene molecule $(6.6 \mathrm{eV})$. Note that this represents an upper limit of the effect due to an idealized equalized charge density distribution over $N$ molecules being used to model the delocalization [16]; a nonuniform distribution leads to less strong shifts.

model described previously [14]. This model was applied here to a canonical OS-pentacene. We elected pentacene because our microelectrostatic model has already been extensively validated against more rigorous quantum-chemical approaches and experimental measurements of pentacene $[14,15]$. Also, the charge transfer integrals are well established by quantumchemical calculations. Finally, pentacene adopts a layered structure in which molecules are oriented nearly vertically on a substrate in a herringbone configuration $[12,16]$. As the transfer integral for holes between adjacent molecular layers is negligible [12], the delocalization is envisaged by dividing a charge equally over $N$ nearest-neighbor molecules within a single layer. We assess the likely delocalization geometry [Fig. 1(a)] based on pentacene charge transfer integrals. These reflect the probability of charge extension over several molecules, which strongly depends on the mutual molecular orientation. The computational details are given elsewhere [17] (see Sec. 1 of the Supplemental Material for more details [18]).

A remarkable finding of these calculations is that the polarization energy $P$, which impacts the position of the conduction/valence band, is decreasing with the extent of charge delocalization [Fig. 1(b)]. This is in line with the recently revealed decreasing trend of $P$ with increasing number of aromatic rings in linear oligoacenes [19]. Delocalizationinduced drop of $P$ and consequent gradual shift of the hole transport energies $E_{h}$ is shown in Fig. 1(c). It represents an upper limit of the effect due to an idealized uniform charge density distribution over neighboring molecules that was used to simulate the charge delocalization [16]. Consequently, $E_{h}$ gradually shifts towards the highest occupied molecular orbital of a single pentacene molecule $(6.6 \mathrm{eV})$ as the charge is delocalized over an increasing number of neighboring molecules. Similar convergence of the transport levels for delocalized charges towards the levels of neutral molecules has been demonstrated by charge modulation spectroscopy on some pentacene derivatives [20]. The calculation above suggests that the position of the valence and conduction band edges in OSs can change notably with the charge delocalization length. Imperfections of crystal structure (disorder) inevitably limit the charge delocalization length, and their nonuniform distribution over a nonperfect crystalline film should give rise to delocalization lengths that are spatially distributed, thereby modulating the transport band edge. 


\section{EMA CHARGE TRANSPORT MODEL}

To assess the effect of the disorder-induced spatial distribution of the charge delocalization length on the charge-carrier transport, we employ the EMA model [21] which we recently suggested. This model is based on the concept of static potential fluctuations in the transport band. The potential fluctuations' concept was originally suggested for imperfect semiconductors [22,23] and it represents an alternative to more common band-tail state descriptions of the charge transport in chalcogenide glasses [24], amorphous oxides [25], and $\alpha-\mathrm{Si}: \mathrm{H}$ [26]. This model was formulated for zero electric field [21]. In this paper, we extend it to arbitrary fields and 2D delocalized transport, and apply it to highly ordered OSs.

The key assumption of our model is that variation of the transport band edge $\varepsilon_{m}$ obeys a Gaussian distribution with the standard deviation $\delta_{0}$ in terms of the Thomas-Fermi approximation [22]: $P\left(\varepsilon_{m}\right)=1 / \delta_{0} \sqrt{2 \pi} \exp \left[-1 / 2\left(\varepsilon_{m} / \delta_{0}\right)^{2}\right]$. These spatial variations are assumed to be sufficiently smooth, with the characteristic length $b$ to be larger than the mean free path of a charge carrier, but much smaller than the length of the system. At low trap density in organic crystalline films, the charge transport can be controlled by the above variations of $\varepsilon_{m}$. The latter constitute potential barriers in the transport band and the system can be considered as energetically weakly disordered with the "disorder" pertaining here to the delocalized band-energy states. Although the transport pertains to percolation, we use an EMA approach that is more appropriate for weakly disordered systems and tacitly encompasses the percolation phenomena. Our method is based on a self-consistency equation for the effective drift conductivity $\sigma_{e}[27,28]$ :

$$
\left\langle\frac{\sigma\left(\varepsilon_{m}\right)-\sigma_{e}}{\sigma\left(\varepsilon_{m}\right)+(d-1) \sigma_{e}}\right\rangle=0,
$$

where $d$ is the spatial dimension of the considered transport problem. $\langle\cdots\rangle$ denotes the configuration averaging. The effective drift mobility $\mu_{e}$ in extended states is then calculated using $\sigma_{e}$. Calculation details are presented in Sec. 2 of the Supplemental Material [18].

To account for the presence of the electric field, $E$, we employ here the concept of the so-called effective dimensionality of the percolation paths, $d_{\mathrm{eff}}$, suggested earlier $[29,30]$. We extend this concept to systems with continuous (that is in fact positional) disorder. The basic idea of the effective dimensionality is the following: At low fields the current in a disordered system flows along certain optimal one-dimensional paths determined by the requirement of maximum current flow, and its local direction does not always coincide with the direction of $E$. It may contain detours around low-conductivity spots. With increasing electric field, the current can no longer bypass regions of low conductivity and the percolation paths become less twisted. This yields lower $\mu_{e}$ at not-too-high $E$ since the carriers are forced to run also through poorly conducting elements. The effect can be described in terms of the $d_{\text {eff }}$, which decreases to 1 with increasing $E$ as established by percolation and EMA theories [29,30], and corroborated by numerical calculations [31]. Rigorous EMA calculations for a 2D positionally disordered system yield $[29,30]$

$$
d_{\text {eff }}=\frac{2}{1+\frac{2}{\pi} \tanh ^{2}\left(\frac{\lambda}{2}\right) K\left[\cosh ^{-2}\left(\frac{\lambda}{2}\right)\right]},
$$

where $K(t)$ is the complete elliptic integral of the first kind, $\lambda=e E a / k_{B} T$, and $a$ is the characteristic mesh length. We assume that Eq. (2) can be applied to any disordered system where the effective conductivity $\sigma_{e}$ can be described by percolation formalism. Thus, to extend our model [21] for arbitrary electric field, we substitute the spatial dimension $d$ in Eq. (1) by $d_{\text {eff }}$ given by Eq. (2) and assume $b=a$ [18].

\section{COMPARISON WITH EXPERIMENT AND DISCUSSION}

Next, we test the model developed above by analyzing TOF photoconductivity measurements of single-crystalline layers of the $\mathrm{C}_{8}$-BTBT. As in the case of pentacene, thin films of $\mathrm{C}_{8}$-BTBT consist of stacked layers whose molecules are oriented nearly vertically relative to the substrate and adopt a herringbone configuration within the plane of the layer. The long alkyl chain of $\mathrm{C}_{8}$-BTBT makes the electronic coupling between adjacent layers extremely weak in the $c$ direction. Thus band transport in $\mathrm{C}_{8}$-BTBT is realized along these layers and the transport problem is two dimensional [12,16], which corresponds well to the microelectrostatic calculations done for pentacene. Recent field-induced time-resolved microwave conductivity measurements revealed unprecedentedly high charge mobility ( $>100 \mathrm{~cm}^{2} / \mathrm{V} \mathrm{s}$ at room temperature) in a BTBT derivative associated with the $2 \mathrm{D}$ character of the crystal [32]. Our $\mathrm{C}_{8}$-BTBT films were processed by zone-casting a 0.25 wt $\% \mathrm{C}_{8}$-BTBT:hexane solution, as described elsewhere $[3,18,33]$. The films exhibit an excellent degree of crystallinity with millimeter-size crystal domains (details of crystal film growth and characterization are described in Sec. 3 of the Supplemental Material [18]). Free-electron-like Hall effect has been previously reported on equivalent $\mathrm{C}_{8}$-BTBT films [11]. These highly crystalline layers are therefore ideally suited for studying delocalized transport.

We performed TOF charge mobility measurements in these crystalline $\mathrm{C}_{8}$-BTBT thin films (see Sec. 4 of the Supplemental Material). The TOF technique was preferred since it operates in a low-carrier concentration regime $[7,8]$ were the spacecharge effects are greatly reduced. In contrast to OFETs, TOF measures bulk mobility values that are much less affected by interface defects/dipoles. Since blocking contacts are used in TOF measurements, charge-carrier injection and associated contact-resistance effects on charge mobility are also reduced. The TOF method employed in this study does not rely on a conventional sandwich-type electrode geometry. It rather uses a coplanar-electrode configuration [a schematic view is shown in the inset of Fig. 2(a)] as described in our earlier work [34]. The main advantage of this method is that the charge transport is probed in the lateral direction parallel to the substrate, along the densely packed plane of $\mathrm{C}_{8}$-BTBT in which delocalized transport is realized. This direction also coincides with the conduction channel in OFET and Hall devices [11].

Figure 2(a) shows the set of $I(t)$ curves in a doublelogarithmic representation obtained at $T=323 \mathrm{~K}$ and at bias voltage $V_{b}$ ranging from 50 to $150 \mathrm{~V}$ (top to bottom). 

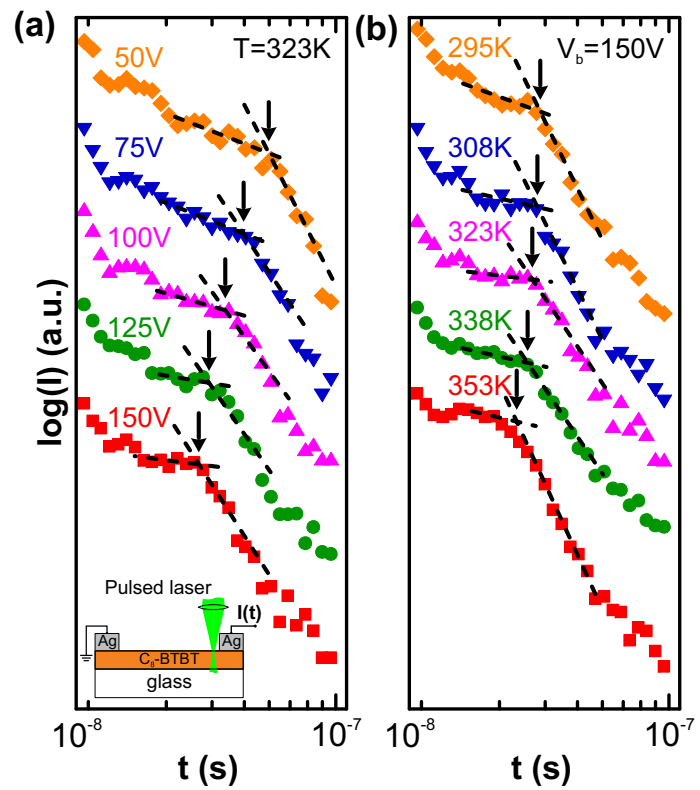

FIG. 2. TOF photocurrent transients measured in $\mathrm{C}_{8}$-BTBT crystalline film at $323 \mathrm{~K}$ under different $V_{b}$ (a) and at constant bias $V_{b}=150 \mathrm{~V}$ for different temperatures (b). Dashed asymptotic lines were used to estimate the transit times (indicated by arrows). Inset in (a) shows a schematic view of the TOF photocurrent measurement.

Figure 2(b) shows $I(t)$ curves obtained at $V_{b}=150 \mathrm{~V}$ and at temperatures ranging from 295 to $353 \mathrm{~K}$ (top to bottom). In order to emphasize the abrupt change in slope of $I(t)$ signaling the charge-carrier transit time $t_{t r}$, we have restricted the time scale to a range from 10 to $100 \mathrm{~ns}$ (the extended time-scale plot of $I(t)$ and experimental details are presented in Sec. 4 of the Supplemental Material [18]). We employed the common method of two asymptotes of different slopes [8] to determine the resulting $t_{t r}$, which is indicated by arrows in Figs. 2(a) and 2(b). In the zeroth-order approximation, the mobility can be determined from $t_{t r}$ using the conventional expression $\mu_{\mathrm{TOF}}=L^{2} / V_{b} t_{t r}$.

The resulting values of $\mu_{\mathrm{TOF}}$ as a function of $V_{b}$ and $T$ are presented in Figs. 3(a) and 3(b), respectively. $\mu_{\mathrm{TOF}}$ reaches values as high as $24 \mathrm{~cm}^{2} / \mathrm{V} \mathrm{s}$ at $T=353 \mathrm{~K}$ and $V_{b}=50 \mathrm{~V}$. This TOF mobility cannot be compared with that obtained in gated experiments, e.g., OFET mobility, which mostly probe a more disordered near-interface crystal layer as compared to bulk. This is because the underlying polar self-assembled monolayer (SAM) dielectric inevitably induces a significant additional electrostatic disorder $(\sim 50-80 \mathrm{meV})[35]$ in the layer near the interface, even if the crystal contains no intrinsic disorder itself. A remarkable observation is that $\mu_{\mathrm{TOF}}$ shows a gradual decrease with applied electric field, reaching $\mu_{\mathrm{TOF}}$ $=12 \mathrm{~cm}^{2} / \mathrm{V}$ s at $T=295 \mathrm{~K}$ and $V_{b}=150 \mathrm{~V}$. At the same time the mobility exhibits a positive temperature dependence $(d \mu / d T>0)$ for all values of $V_{b}$. To the best of our knowledge, no observation of the negative field dependence of mobility (NFDM) for a band-transport organic material in a high-temperature region has been reported before. NFDM has been observed in some hopping-transport disordered solids at low/moderate fields [36-39]. The effect is material specific and
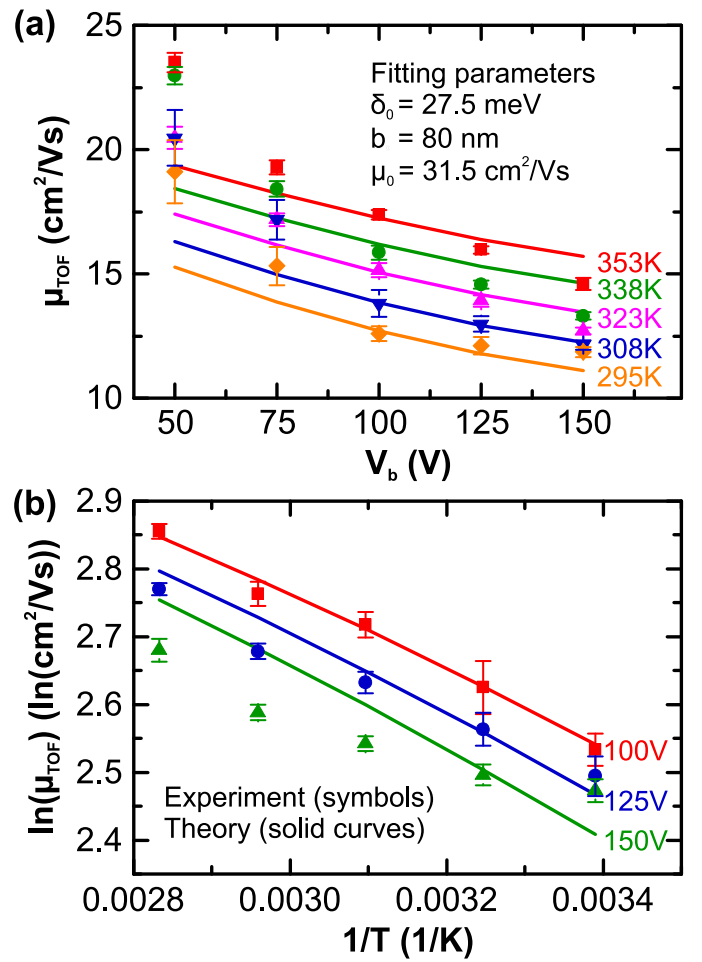

FIG. 3. (a) Field dependences of the TOF mobility measured (symbols) at different temperatures, and (b) the mobility temperature dependences at different $V_{b}$ in $\mathrm{C}_{8}$-BTBT crystalline films. Fitting by the present EMA model is shown by solid curves. Electric field was estimated as $E=V_{b} / L$.

results from an enhanced positional disorder as demonstrated by Monte Carlo simulations [7] and EMA calculations [40]. It is noteworthy that the chevron-shaped morphology of the $\mathrm{C}_{8}$-BTBT crystalline film, as revealed by AFM images in Sec. 3 of the Supplemental Material [18], facilitates the creation of dead ends in percolative paths, corresponding to an enhanced positional disorder. In perfect organic crystals, NFDM is expected only for the hot-charge-carrier motion realized at low temperatures where distinct nonactivated $\mu \propto T^{-n}$ band transport manifests itself, as demonstrated earlier for naphthalene and perylene single crystals $[9,10]$. Our experiments, however, were performed at room temperature and above, where hot-carrier transport can be ruled out. Besides, a possible impact of the diffusion-controlled transport on the observed NFDM is expected at much lower fields $\left(V_{c r}=\right.$ $0.05 \mathrm{~V}$ for our samples) $[18,40]$ and thus can be excluded.

The EMA transport model developed above allows for a quantitative description of our unusual observations. Fitting of the $\mu(E)$ and $\mu(T)$ dependences by the present model is shown in Figs. 3(a) and 3(b) (solid lines), respectively. Some quantitative deviation of the fitting curves can still be noticed for some data points-this is due to a simplified character of the present model and to its ignoring some other relevant phenomena capable of affecting the mobility. For instance, charge-phonon scattering or the effective mass approach was neglected here due to the lack of appropriate theory for it for $\mathrm{C}_{8}$-BTBT. The present study is mainly focused on understanding the key physical mechanism responsible for the 
unusual charge transport behaviors and tendencies observed here; therefore considering the simplicity of the model, the agreement with experimental data might be considered as reasonably good. The only fitting parameters are $\delta_{0}=27.5 \mathrm{meV}$, $b=80 \mathrm{~nm}$, and $\mu_{0}=31.5 \mathrm{~cm}^{2} / \mathrm{Vs}$ (indicated in the inset) and look reasonable. $\delta_{0}$ is close to $\delta_{0}=30 \mathrm{meV}$ estimated by recent first-principles calculations [21] for amorphous oxide semiconductors featuring similarly high delocalized mobility, while it is significantly less than $\delta_{0}=130 \mathrm{meV}$ reported for $\alpha$-Si:H [26] where $\mu$ is much lower. The obtained parameter $b$ is several orders of magnitude less than the channel length $L=75 \mu \mathrm{m}$, which conforms well to the model requirement $b \ll L$. The intrinsic mobility was estimated as $\mu_{0}=$ $31.5 \mathrm{~cm}^{2} / \mathrm{V} \mathrm{s}$, which is in the same region as the band mobility predicted by ab initio calculations for $\mathrm{C}_{8}$-BTBT $[12,41]$.

Finally, it should be noted that, apart from the aboveconsidered long-range static fluctuations, there might also be "dynamic" potential fluctuations due to the time evolution and correlations of the charge localization strength as recently demonstrated for perfect organic single crystals by quantumchemical approaches $[20,42,43]$. It would thus appear plausible that, in an imperfect organic system with a (weak) static disorder, the long-range band edge variations might arise from a superposition of both static and dynamic components of the potential fluctuations. However, the dynamic potential fluctuations alone are fundamentally unable to give rise to the NFDM observed at low/moderate fields together with positive mobility temperature dependence in our highly crystalline, yet imperfect, $\mathrm{C}_{8}$-BTBT films. Treatment of the NFDM effect in terms of the field-induced transformation of percolation paths is meaningful only for a disordered system with "static" energy landscape variations. To the best of our knowledge, there is no theoretical model available which could rationalize the NFDM effect based on the "dynamic" disorder which is hardly compatible with established percolation theories. Nonetheless, the presence of dynamic potential fluctuations could contribute to the temperature dependence of the mobility; however, it is not expected to alter qualitatively our major findings. Extension of the present theoretical formalism to account for the dynamic disorder is beyond the scope of the present study. We believe that since the present model captures the observed (quite nontrivial) experimental tendencies correctly, this justifies its applicability at this stage.

In conclusion, we have found that, in contrast to inorganic semiconductors, the energy level structure of organic semiconductors depends significantly on the degree of charge delocalization. Spatial distribution of the charge delocalization length can cause long-range potential fluctuations of the band edge in crystalline films. This is an alternative concept for organic semiconductors and we invoke it to formulate an analytic model for the charge transport through delocalized states in organic crystalline films. The model was applied to highmobility $\mathrm{C}_{8}$-BTBT thin crystalline films with a pronounced 2D charge delocalization behavior. The observed negative field dependence (NFDM) coupled with positive temperature dependence of the hole mobility measured in these crystalline films by a lateral TOF method can naturally be described by the present model. The NFDM stems from predominance of a positional disorder. The suggested mechanism can have important implications for description of the charge transport via delocalized states in weakly disordered high-mobility OSs with negligible density of localized states. The principal advantage of the present model is that it can solve the puzzling observation of positive $\mu(T)$ dependence for the charge transport via delocalized states for both drift and Hall mobilities reported earlier in the literature for band-transport materials [20,44].

\section{ACKNOWLEDGMENTS}

The authors thank Nippon Kayaku Co. for providing $\mathrm{C}_{8}$-BTBT for this study. This research was financially supported by the Slovenian Research Agency and the Belgian FWO for financial support in the framework of the international collaboration project ORSIC-HIMA (Project No. N1-0024/G0B5914N). Additional funding was received from the European Research Council under the European Union's Seventh Framework Programme (FP7/2007-2013)/ERC Grant Agreement No. 320680 (EPOS CRYSTALLI), from the SRA's Program P1-0055, and from the Volkswagen Foundation through the project "Understanding the dependence of charge transport on morphology in organic semiconductor films".

A.K., F.T., and R.J. contributed equally to this work.
[1] T. Uemura, Y. Hirose, M. Uno, K. Takimiya, and J. Takeya, Appl. Phys. Express 2, 111501 (2009).

[2] H. A. Becerril, M. E. Roberts, Z. Liu, J. Locklin, and Z. Bao, Adv. Mater. 20, 2588 (2008).

[3] R. Janneck, F. Vercesi, P. Heremans, J. Genoe, and C. Rolin, Adv. Mater. 28, 8007 (2016).

[4] H. Ebata, T. Izawa, E. Miyazaki, K. Takimiya, M. Ikeda, H. Kuwabara, and T. Yui, J. Am. Chem. Soc. 129, 15732 (2007)

[5] T. Yamamoto and K. Takimiya, J. Am. Chem. Soc. 129, 2224 (2007).

[6] V. Coropceanu, J. Cornil, D. A. da Silva Filho, Y. Olivier, R. Silbey, and J. L. Brédas, Chem. Rev. 107, 926 (2007).

[7] H. Bässler, Phys. Status Solidi 175, 15 (1993).

[8] P. M. Borsenberger and D. S. Weiss, Organic Photoreceptors for Xerography (Dekker, New York, 1998).
[9] W. Warta and N. Karl, Phys. Rev. B 32, 1172 (1985)

[10] N. Karl, Synth. Met. 133-134, 649 (2003).

[11] T. Uemura, K. Nakayama, Y. Hirose, J. Soeda, M. Uno, W. Li, M. Yamagishi, Y. Okada, and J. Takeya, Curr. Appl. Phys. 12, S87 (2012).

[12] H. Kobayashi, N. Kobayashi, S. Hosoi, N. Koshitani, D. Murakami, R. Shirasawa, Y. Kudo, D. Hobara, Y. Tokita, and M. Itabashi, J. Chem. Phys. 139, 014707 (2013).

[13] E. V. Emelianova, S. Athanasopoulos, R. J. Silbey, and D. Beljonne, Phys. Rev. Lett. 104, 206405 (2010).

[14] S. Verlaak, D. Beljonne, D. Cheyns, C. Rolin, M. Linares, F. Castet, J. Cornil, and P. Heremans, Adv. Funct. Mater. 19, 3809 (2009).

[15] A. Mityashin, Y. Olivier, T. Van Regemorter, C. Rolin, S. Verlaak, N. G. Martinelli, D. Beljonne, J. Cornil, J. Genoe, and P. Heremans, Adv. Mater. 24, 1535 (2012). 
[16] J. Xi, M. Long, L. Tang, D. Wang, and Z. Shuai, Nanoscale 4, 4348 (2012).

[17] S. Verlaak and P. Heremans, Phys. Rev. B 75, 115127 (2007).

[18] See Supplemental Material at http://link.aps.org/supplemental/ 10.1103/PhysRevB.96.125202 for more details about molecular microelectrostatic model calculation of the transport energy level upon the degree of the charge delocalization (Sec. 1), EMA transport model formulations (Sec. 2), crystal film growth and characterization (Sec. 3), and TOF photoconductivity experiments (Sec. 4).

[19] G. D’Avino, L. Muccioli, C. Zannoni, D. Beljonne, and Z. G. Soos, J. Chem. Theory Comput. 10, 4959 (2014).

[20] J.-F. Chang, T. Sakanoue, Y. Olivier, T. Uemura, M.-B. Dufourg-Madec, S. G. Yeates, J. Cornil, J. Takeya, A. Troisi, and H. Sirringhaus, Phys. Rev. Lett. 107, 066601 (2011).

[21] I. I. Fishchuk, A. Kadashchuk, A. Bhoolokam, A. de Jamblinne de Meux, G. Pourtois, M. M. Gavrilyuk, A. Köhler, H. Bässler, P. Heremans, and J. Genoe, Phys. Rev. B 93, 195204 (2016).

[22] E. O. Kane, Phys. Rev. 131, 79 (1963).

[23] B. I. Shklovskii and A. L. Efros, Electronic Processes of Doped Semiconductors (Springer-Verlag, Berlin, 1984).

[24] M. Nardone, M. Simon, I. V. Karpov, and V. G. Karpov, J. Appl. Phys. 112, 071101 (2012).

[25] T. Kamiya, K. Nomura, and H. Hosono, Appl. Phys. Lett. 96, 122103 (2010).

[26] J. A. Howard and R. A. Street, Phys. Rev. B 44, 7935 (1991).

[27] S. Kirkpatrick, Rev. Mod. Phys. 45, 574 (1973).

[28] I. I. Fishchuk, A. Kadashchuk, S. T. Hoffmann, S. Athanasopoulos, J. Genoe, H. Bässler, and A. Köhler, Phys. Rev. B 88, 125202 (2013).

[29] H. Böttger and V. V Bryksin, Phys. Status Solidi 96, 219 (1979).
[30] H. Böttger and V. V. Bryksin, Phys. Status Solidi 113, 9 (1982).

[31] H. Böttger and D. Wegener, Philos. Mag. B 50, 409 (1984).

[32] Y. Tsutsui, G. Schweicher, B. Chattopadhyay, T. Sakurai, J.-B. Arlin, C. Ruzié, A. Aliev, A. Ciesielski, S. Colella, A. R. Kennedy, V. Lemaur, Y. Olivier, R. Hadji, L. Sanguinet, F. Castet, S. Osella, D. Dudenko, D. Beljonne, J. Cornil, P. Samorì et al., Adv. Mater. 28, 7106 (2016).

[33] W. Pisula, A. Menon, M. Stepputat, I. Lieberwirth, U. Kolb, A. Tracz, H. Sirringhaus, T. Pakula, and K. Müllen, Adv. Mater. 17, 684 (2005).

[34] E. Pavlica and G. Bratina, Appl. Phys. Lett. 101, 093304 (2012).

[35] A. Mityashin, O. M. Roscioni, L. Muccioli, C. Zannoni, V. Geskin, J. Cornil, D. Janssen, S. Steudel, J. Genoe, and P. Heremans, ACS Appl. Mater. Interfaces 6, 15372 (2014).

[36] P. M. Borsenberger, L. Pautmeier, and H. Bässler, J. Chem. Phys. 94, 5447 (1991).

[37] A. J. Mozer, N. S. Sariciftci, A. Pivrikas, R. Österbacka, G. Juška, L. Brassat, and H. Bässler, Phys. Rev. B 71, 035214 (2005).

[38] M. Guo, X. Yan, and T. Goodson, Adv. Mater. 20, 4167 (2008).

[39] S. R. Mohan, M. P. Joshi, and M. P. Singh, Chem. Phys. Lett. 470, 279 (2009).

[40] I. I. Fishchuk, A. Kadashchuk, H. Bässler, and M. Abkowitz, Phys. Rev. B 70, 245212 (2004).

[41] W. Shi, J. Chen, J. Xi, D. Wang, and Z. Shuai, Chem. Mater. 26, 2669 (2014).

[42] L. Wang and D. Beljonne, J. Phys. Chem. Lett. 4, 1888 (2013).

[43] A. A. Johansson and S. Stafström, Phys. Rev. B 69, 235205 (2004).

[44] S. Haas, A. F. Stassen, G. Schuck, K. P. Pernstich, D. J. Gundlach, B. Batlogg, U. Berens, and H.-J. Kirner, Phys. Rev. B 76, 115203 (2007). 